\title{
Ayotzinapa. La incansable lucha por la verdad, la justicia y la vida
}

En el contexto del segundo aniversario de la aparición del movimiento de Ayotzinapa, fue publicado el libro Ayotzinapa. La incansable lucha por la verdad, la justicia y la vida, que es una continuación de uno anterior, Ayotzinapa. Una fuerte indignación que se convirtió en movimiento. El nuevo libro avanza y profundiza la reflexión sobre las características de este movimiento social y sobre cómo se han ido abandonando aspectos que dieron vida a otros movimientos sociales en distintos momentos y con otras demandas.

En el primer libro, los autores exploraron la relación entre el movimiento de los padres de los normalistas y el Movimiento por la Paz con Justicia y Dignidad, mientras que en este segundo libro profundizan en las posibilidades abiertas por Ayotzinapa para aglutinar diversas demandas de manera solidaria y con la construcción de acuerdos comunes, muy en la línea de lo que los zapatistas les dijeron a los padres de los normalistas a finales del 2014, sobre que siguieran buscando otros dolores y otras rebeldías, unidas en una misma resistencia contra el capitalismo depredador.

En este segundo libro, por su parte, se exploran

Carlos Alonso Reynoso, y Jorge Alonso Sánchez (2016). Ayotzinapa. La incansable lucha por la verdad, la justicia y la vida. Guadalajara: Universidad de Guadalajara. 
las expresiones de este novedoso movimiento desde finales de 2014 hasta mediados de mayo de 2016, cuando inició un periodo intenso de lucha magisterial. Una primera característica del movimiento de Ayotzinapa mencionada por los autores es que "ha tenido una intensidad que no se le había visto a otros movimientos de este tipo" (p. 10), por lo que ellos se proponen "comprender lo que lo hace específico. Ha conmovido profundamente a México y al mundo. Tiene un objetivo vital que lo hace existir y ser y un componente de lograr ser oportunidad para la convergencia de muchas luchas" (p. 10). Como los mismos autores reconocen, sobre el segundo punto se han hecho algunos ensayos.

Para ubicar mejor estas expresiones de un movimiento social como el de Ayotzinapa, los autores inician el libro con un capítulo que recoge de manera sintética la irrupción del movimiento y de su acción inicial. En breves páginas tenemos una visión de conjunto de la manera en que se ha ido construyendo este incansable movimiento que ha sabido trasmitir su dolor, rabia y rebeldía y, en esa medida, ha sido capaz de unificar. De manera paralela y altamente contrastante, los autores van comparando la actuación de las autoridades (la "inconsistente versión oficial", según la llaman), desde la construcción de la mal llamada "versión histórica" hasta su desmentido contundente con la investigación del Grupo Interdisciplinario de Expertos Independientes (GIEI) de la Comisión Interamericana de Derechos Humanos (CIDH), creado tras las presiones a nivel nacional e internacional, aceptado y financiado por el Gobierno federal y, posteriormente, expulsado por el propio Gobierno.

El segundo capítulo se centra en la actuación del GIEI, en buena medida porque se trata de la intervención de un organismo externo que se enfrentó, desde el inicio de su trabajo, con la oposición de diversos funcionarios del Gobierno, incluso con el trabajo sucio de periodistas y columnistas mercenarios. El análisis realizado en el libro no pierde 
ningún detalle, revisa todas las oposiciones, desde la que se da entre expertos, peritos y universidades, hasta las de los centros de investigación.

El papel de los forenses argentinos resulta fundamental. La conclusión del GIEI, desde su primer informe y luego consolidada en el segundo, significa la destrucción de la "verdad histórica" (que quedó como lo que realmente es, una gran mentira). Además, el nuevo libro retoma las diversas líneas de investigación sugeridas por el GIEI -y que no han sido retomadas-, como el papel relevante, por omisión, del $27^{\circ}$ Batallón de Infantería, y la indagación sobre el quinto camión, presumiblemente cargado de droga con destino a Chicago.

El tercer capítulo documenta todo el proceso de encuentros y desencuentros del GIEI con funcionarios gubernamentales, y de eso hasta la decisión de no prorrogar su estancia en el país contra la voluntad y propuesta del movimiento de Ayotzinapa y diversas ONG que los han acompañado, las cuales solicitaban la permanencia del GIEI por tiempo indefinido hasta que se diera con el paradero de los normalistas.

Dada la riqueza de este movimiento, que ha dado mucho qué pensar debido a su persistencia, intensidad y, sobre todo, por ser incansable, como señalan los autores, estos dedican un cuarto capítulo a recoger algunas opiniones que se han vertido a lo largo de este proceso: libros, capítulos de libro, columnas de opinión, cualquier fuente de información en torno al movimiento Ayotzinapa. De este conjunto, los autores hacen un balance y ofrecen "las pistas que encontramos para comprender un movimiento tan rico y complejo" (p. 11).

El final de la introducción de este libro es la principal clave de su lectura: "Una pregunta rectora de la investigación ha sido si el movimiento de Ayotzinapa pudiera encuadrarse en uno más de los movimientos de indignados o si aportaba elementos que lo hicieran distinto" (p. 11). 
No deja de llamar la atención que el libro, como muchos otros, no incluya conclusiones sino un apartado llamado "Para seguir reflexionando", dentro del cual un primer punto de interés es una afirmación de los autores: "Consideramos que no es un movimiento social más [...], preferimos situar al movimiento de Ayotzinapa como un movimiento de otra índole" (p. 211).

Luego de hacer un breve recuento de las teorías sobre los movimientos sociales (desde los clásicos, con carácter de clase, o los nuevos, de carácter sectorial, o incluso los que se han generado en estos diecisiete años del siglo XXI, algunos que expresan malestares y algunos que realizan propuestas), los autores prefieren

[...] llamar la atención de las tendencias de análisis para las cuales la terminología de movimiento social no es suficiente para dar cuenta de la fortaleza de ciertos acontecimientos en donde se pueden detectar identidades o la utilización de determinados repertorios en ciertos espacios de oportunidad, pero en lo que eso no llega a ser lo central (p. 2I2).

Además de la crítica hacia las teorías clásicas de los movimientos sociales o de los nuevos movimientos, este libro nos advierte sobre un criterio para caracterizar a los movimientos sociales más recientes, surgidos en diversas partes del mundo: "se ubican dentro de los límites del capital y del Estado, pues dentro del poder del capitalismo no es posible que se hagan cambios en contra de las relaciones que aquellos [capital y Estado] generan" (p. 212).

Con este criterio, se intenta ubicar a los movimientos emancipatorios, caracterizados por la organización autónoma para la construcción de una sociedad diferente y de relaciones que no sean capitalistas. Aquí es donde los autores ubican al movimiento Ayotzinapa: 
[...] no tanto entre los movimientos sociales, sino en los que tienen dinámicas emancipatorias, en los que desde abajo buscan liberarse del dominio de los de arriba. Aunque existe el peligro de que los de arriba aprendan a prevenir y combatir eficazmente la respuesta popular, también no habría que perder de vista que las luchas de los de abajo también han ido sacando lecciones de cómo irse defendiendo ante viejos y nuevos embates (p. 2/3).

Esta interpretación se apoya en la opinión de Gustavo Esteva y Raúl Zibechi, para quienes el movimiento Ayotzinapa no es un movimiento que se proponga reconstruir un mundo que les arrebató a sus hijos, sino un mundo totalmente otro, nuevo, pues en los hilos que ha ido tejiendo Ayotzinapa se detecta un impulso en la lógica de la resistencia y la rebeldía.

De esta manera, los autores van sumando características, algunas retomadas de los movimientos tradicionales o de los más recientes, que hacen explícita, por ejemplo, la lucha por las mentes, pues los movimientos enfrentan como una de sus más duras batallas que los grandes medios de comunicación y periodistas mercenarios al servicio del poder traten de imponer una visión negativa del movimiento y hasta animen a sus integrantes a que, como el presidente Peña les diría, "ya superen su problema".

Esa conquista de mentes implica visibilizar todos los dolores y sufrimientos provocados por la violencia del crimen hecho Estado, que no es sino una guerra abierta contra el pueblo. Todas las fechas de celebración, cívicas y religiosas, son para expresar la lucha del movimiento de Ayotzinapa, estrechamente ligado al 02 de octubre, porque para esa fecha ya había ocurrido la trágica desaparición de los normalistas. Por ello, otros movimientos los invitan a que encabecen sus marchas. Los autores destacan que el movimiento de Ayotzinapa 
[...] ha sido un movimiento que ha hecho suyas luchas legítimas del pueblo, $y$ en sus múltiples caravanas por el país ha ido abrazando todas las luchas populares que ha contactado intentando que no haya luchas aisladas. No hegemoniza las luchas, sino que busca formas fluidas de convergencias (p. 216).

Los zapatistas han llamado a su lucha una guerra contra el olvido. Ante el movimiento de Ayotzinapa, el Gobierno apuesta al olvido, y el movimiento, por su parte, a la memoria de los 43 y de los miles de desaparecidos. Esa memoria es la que mantiene la lucha por la presentación con vida de los 43 y los miles de desaparecidos, la demanda de justicia y en contra de los crímenes de Estado; exige el cumplimiento de las recomendaciones de relatores especiales y comités de expertos de la ONU, de la CIDH y, sobre todo, del GIEI. A pesar de todo, y de las más recientes publicaciones sobre la investigación interna de la Procuraduría General de la República (PGR), el Gobierno insiste en su mentira.

Uno de los aspectos que más llaman la atención sobre el movimiento Ayotzinapa tiene que ver con esa lucha por las mentes, una verdadera batalla simbólica, la cual tuvo un fuerte enfrentamiento en la pasada visita del papa Francisco: ganó el Estado al evitar el encuentro del papa con los padres de los normalistas, sin embargo, el mismo Estado pierde la batalla por la verdad con los informes del GIEI, de los forenses argentinos y de innumerables ONG defensoras de los derechos humanos que acompañan al movimiento Ayotzinapa.

Contra quienes afirman que el movimiento Ayotzinapa se quedó en sus 43 desaparecidos, el movimiento ha hecho

[...] todo lo contrario: apelando a sus 43 simbólicamente siempre ha enfatizado los miles de desaparecidos; pero no quiso reducirse a un movimiento más de víctimas. Ante la muerte desatada, es un movimiento 
que proclama la vida. El movimiento de Ayotzinapa se ha convertido en un movimiento eminentemente emancipatorio (p. 219).

En el análisis pormenorizado de este libro, resalta el proceso organizativo del movimiento Ayotzinapa, desde el comité de padres y madres de los 43 , el comité estudiantil de la Normal, el Congreso Nacional Indígena (CNI) y la Sexta Nacional e Internacional, la Asamblea Nacional Popular (ANP) y un conjunto de colectivos y organizaciones que se han solidarizado con esta lucha. De esta manera surgen las acciones globales de cada día 26 y se impulsan innumerables acciones organizativas a nivel local y regional.

Los autores reconocen que la mayor fortaleza del movimiento Ayotzinapa ha sido y sigue siendo la construcción desde abajo de redes y organizaciones para ir propiciando que se vaya delineando un México totalmente otro. Sin desconocer las contradicciones que hay en el movimiento, se trata de un movimiento permanente, que no se ha quebrantado $\mathrm{y}$, como dicen los zapatistas, no se vende, no claudica y se mantiene rebelde e indignado.

Finalmente, en su relación de hijo y padre, los autores nos recuerdan el papel de los sentimientos y las emociones en la construcción de este movimiento, pues afirman:

El amor de los padres por sus hijos desaparecidos fue el motor para una lucha dura inspirada por la esperanza de la vida. Prosigue consistentemente en la lucha por la vida. Pasó de ser un movimiento de desaparecidos a uno que busca insistentemente la transformación del país. La lucha del movimiento ha sido por la verdad, la justicia, la vida y por el derecho de los de abajo a vivir dignamente (p. 225).

Así concluye este libro, cuya lectura apasiona. 